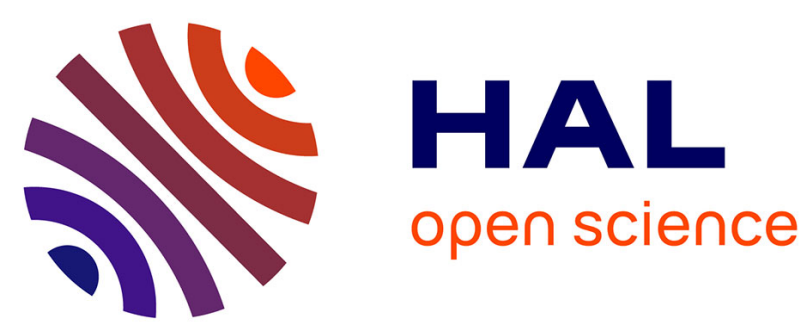

\title{
Development of a fluidic cell to image precipitation reactions by x-ray microscopy
}

Charlie Gosse, Joni Frederick, Christian Blot, Stephane Lefrancois, Sufal Swaraj, Stefan Stanescu, Rachid Belkhou, Patrick Guenoun, Corinne

Chevallard

\section{To cite this version:}

Charlie Gosse, Joni Frederick, Christian Blot, Stephane Lefrancois, Sufal Swaraj, et al.. Development of a fluidic cell to image precipitation reactions by x-ray microscopy. 2017 19th International Conference on Solid-State Sensors, Actuators and Microsystems (TRANSDUCERS), Jun 2017, Kaohsiung, Taiwan. pp.1734-1737, 10.1109/TRANSDUCERS.2017.7994402 . cea-02368479

\section{HAL Id: cea-02368479 https://hal-cea.archives-ouvertes.fr/cea-02368479}

Submitted on 18 Nov 2019

HAL is a multi-disciplinary open access archive for the deposit and dissemination of scientific research documents, whether they are published or not. The documents may come from teaching and research institutions in France or abroad, or from public or private research centers.
L'archive ouverte pluridisciplinaire HAL, est destinée au dépôt et à la diffusion de documents scientifiques de niveau recherche, publiés ou non, émanant des établissements d'enseignement et de recherche français ou étrangers, des laboratoires publics ou privés. 


\title{
DEVELOPMENT OF A FLUIDIC CELL TO IMAGE PRECIPITATION REACTIONS BY X-RAY MICROSCOPY
}

\author{
Charlie Gosse ${ }^{1}$, Joni Frederick², Christian Blot ${ }^{2}$, Stéphane Lefrançois ${ }^{3}$, Sufal Swaraj ${ }^{4}$, \\ Stefan Stanescu, ${ }^{4}$ Rachid Belkhou ${ }^{4}$, Patrick Guenoun ${ }^{2}$, and Corinne Chevallard ${ }^{2}$ \\ ${ }^{1}$ Laboratoire de Photonique et de Nanostructures, LPN-CNRS, Marcoussis, FRANCE \\ ${ }^{2}$ LIONS, IRAMIS, CEA-Saclay, Gif-sur-Yvette, FRANCE \\ ${ }^{3}$ SMIS beamline, SOLEIL synchrotron, Saint-Aubin, FRANCE \\ ${ }^{4}$ HERMES beamline, SOLEIL synchrotron, Saint-Aubin, FRANCE
}

\begin{abstract}
We introduce an observation chamber dedicated to image, at the nanoscale, the kinetics of reactions taking place at the liquid/solid interface. The assembly of this microfluidic cell is straightforward and, most importantly, its design allow the operator to precisely regulate the hydrodynamic flow and control the concentration in reagents at the observation window.
\end{abstract}

\section{KEYWORDS}

Chemical kinetics, biomineralization, scanning transmission X-ray microscopy, in operando imaging, microreactor.

\section{INTRODUCTION}

Biominerals made of calcium carbonate are formed of $\sim 100 \mathrm{~nm}$ large nanoparticles, the crystallization and the self-assembly of which are controlled by natural organic molecules. With respective spatial and temporal resolutions around $20 \mathrm{~nm}$ and $1 \mathrm{~min}$, respectively, scanning transmission X-ray microscopy (STXM) is thus the technique of choice to unravel the early steps of material growth [1]. However, because photons in this energy range are readily absorbed, observations must be performed under vacuum and on micron-thin samples: two requirements that become challenging when operating with solutions and when trying to decipher chemical dynamics on the minute time-scale.

In situ observations are often performed in a wet cell where the liquid sample is sandwiched in between two SiN membranes fabricated on silicon frames. This chamber is then introduced in a holder closed by a lid, the whole being made vacuum-tight thanks to O-rings. However, such protocol do not allow one to catch the early events of a precipitation reaction since at least 20 to $30 \mathrm{~min}$ are necessary to load the sample, pump the microscope chamber, and focus the optics.

Thus, the observation chamber need to be connected to capillaries dedicated to convey, as quickly as possible, the reactive mixture under the beam (Fig. 1,2). One of the requirements is now to precisely control the fluid flow without any leak. Sealing the two silicon frames during microfabrication [2,3] is optimal from a fluidic point of view; nevertheless, it impedes any of the "just-beforeuse" surface treatments often realized to facilitate filling and observation. Conversely, some commercial products rely on semi-closed observation chamber obtained by non-covalent assembly [4,5] (S-setup, Fig. 2b-b'). However, in these solutions large cavities are present around the silicon chips, which hinder any fine regulation of the flow of reagents over the SiN windows. Thanks to especially designed elastomeric gaskets, we here propose a technique enabling to precisely control fluid actuation while still making use of chips simply brought into contact (F-setup, Fig. 2a-a').
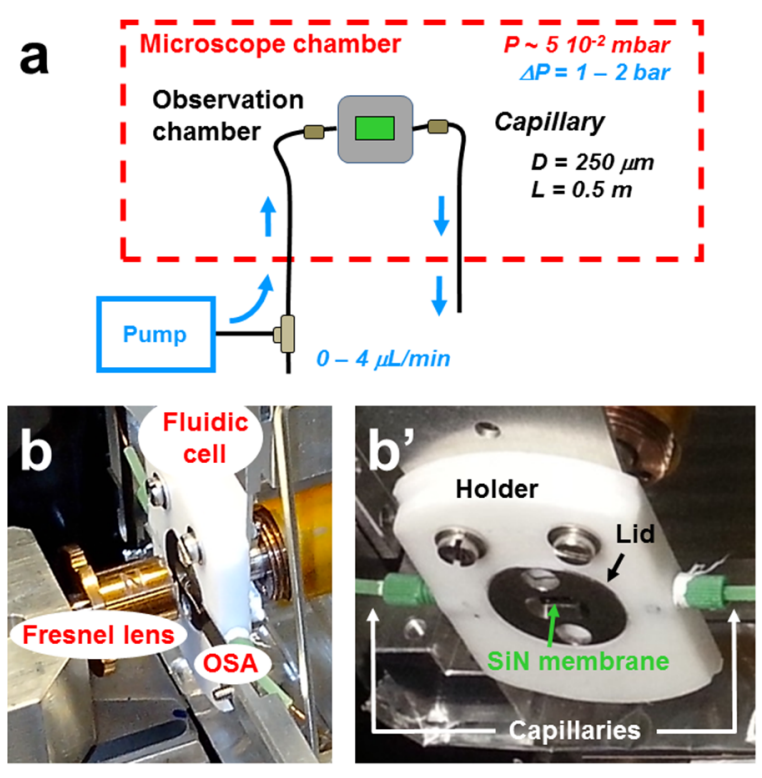

Figure 1: Setup layout. (a) Diagram of the fluidic system. (b-b') Picture of the fluidic cell mounted on the PolLux STXM.

\section{EXPERIMENTAL SECTION}

\section{Scanning transmission X-ray microscopy}

Imaging and spectrocospy were achieved on the STXM setups located on the PolLux beamline of the Swiss Light Source (SLS, Villigen, Swiss) and on the HERMES beamline of the SOLEIL synchrotron (SaintAubin, France). Both microscopes were fabricated by RI Research Instruments (Bergisch Gladbach, Germany). They follow a similar design that combines coarse and fine interferometer controlled mechanical stages, which insures scanning capabilities with a nanometer resolution. Nevertheless, the STXM at HERMES has the advantage of more space available around the sample, which is of huge interest when using complex environments like the present fluidic cell. During operation vacuum inside the microscope chamber is always lower than $5 \times 10^{-2} \mathrm{mbar}$.

Data were typically recorded in the "water window", i.e. between 280 and $530 \mathrm{eV}$. In this energy range micronthick water films are transparent while nitrogen, carbon, 
and calcium - elements that are largely present in biological specimens - are absorbing. At $510 \mathrm{eV}$ the linear attenuation coefficients for silicon nitride and for water are 4.08 and $0.10 \times 10^{4} \mathrm{~cm}^{-1}$, respectively. A $50 \mathrm{~nm}$ thick observation window and a $3 \mu \mathrm{m}$ thick aqueous sample will thus transmit 81 and $74 \%$ of the incident photons, respectively. At $350 \mathrm{eV}$ these values are roughly twice lower.

Diffractive optics (i.e. a Fresnel zone plate) is used to focus the X-ray beam down to $30-40 \mathrm{~nm}$. Focal distances at these energies are in the few millimeters range. As a consequence, only 5 to $10 \mathrm{~mm}$ are available to position the order sorting aperture (a thin hollow metal plate that filters the first diffraction order) and the fluidic cell along the optical axis, between the zone plate and the detector (a photomultiplier).

STXM data were recorded relying on two different measurement schemes, either imaging was achieved by scanning the sample at fixed energy or spectra were acquired over limited spatial areas by tuning the energy through an absorption edge.
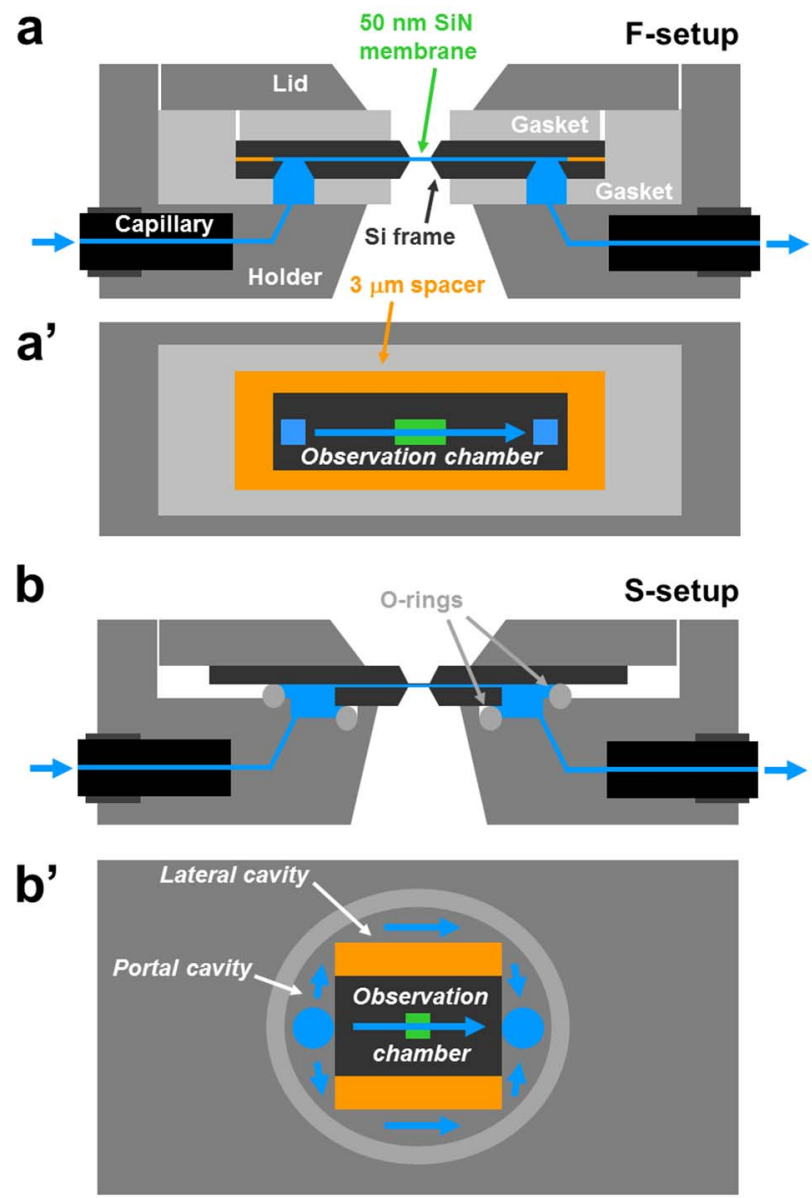

Figure 2: Diagrams for two fluidic cell designs. (a-a') Our fully closed observation chamber, the F-setup. (b-b') An example of semi-closed observation chamber, the $S$ setup. $(a, b)$ Cross-sectional views along the optical axis. $\left(a^{\prime}, b^{\prime}\right)$ Top views with the lid and the top chip removedfor the sake of clarity the flow of water over the bottom chip and over the holder is only represented with blue arrows.

\section{Microfluidics}

Suspended SiN membranes, $50 \mathrm{~nm}$ thick and $100 \mu \mathrm{m}$ $\times 300 \mu \mathrm{m}$ large, were fabricated on a silicon frame, 200 $\mu \mathrm{m}$ thick and $3 \mathrm{~mm} \times 6 \mathrm{~mm}$ large, by Norcada (Edmonton, Canada). A rectangular $\mathrm{SiO}_{2} / \mathrm{SiN}_{\mathrm{x}}$ spacer was additionally deposited on one of the chip, in order to define a $1.5 \mathrm{~mm} \times 5 \mathrm{~mm}$ observation chamber. Two access holes dedicated to fluidic actuation were also obtained by breaking two lateral, $100 \mu \mathrm{m} \times 100 \mu \mathrm{m}$, membranes (Fig. 2a-a').

Mounting the fluidic cell was simply achieved by treating the membranes with an oxygen plasma to favour wetting, positioning them on the top of each other in between home-made elastomeric gaskets, and pressing the whole assembly in between a Teflon holder and a stainless steel lid (Fig. 1b-b',2a-a'). PEEK or FPE capillaries, with a $250 \mu \mathrm{m}$ inner diameter and an $80 \mathrm{~cm}$ length, as well as PEEK screws (Upchurch Scientific) were next used to connect the fluidic pumping system (Fig. 1). More precisely, actuation was driven by a MFCS-EZ controller from Fluigent (Villejuif, France) located outside of the microscope chamber. The flow rate at the entrance and at the exit of the microscope was measured with two flowmeters, which enabled us to check for any mass imbalance, and thereby to detect possible leaks.

\section{Hydrodynamics modeling}

In order to evaluate how reagents are transported from the reservoirs to the observation window we built coarse-grain "resistor models" of the Figure 2 fluidic setups (Fig. 3). For each compartment, the pressure difference between its outlet and its inlet, $\Delta P$, is related to the corresponding flowrate, $Q$, by an equivalent of the Ohm's law: $\Delta P=R \times Q$, with $R$ the hydrodynamic resistance. More specifically, for a channel of length $L$ one has $R=\left[12 \mu L / W H^{3}\right] \times\left[1-192 H / \pi^{5} W\right]^{-1}$ when the cross-section is a rectangle of width $W$ and height $H$ [6], and $R=128 \mu L / \pi D^{4}$ when the cross-section is a circle of diameter $D$ [7]. The dynamic viscosity of the fluid, $\mu$, was here assumed to be the one of water, i.e. $10^{-3} \mathrm{~Pa}$.s. Note also that in first approximation we did not take into account the bulging of the $\mathrm{SiN}$ membranes in the computation of $R$ for the observation chamber.

Using composition rules similar to those of electrokinetics, the hydrodynamic resistances for the whole setups could be calculated: $R_{F}=2 R_{C}+R_{O C} \approx R_{O C}$ and $R_{S}=2\left(R_{C}+R_{P C}\right)+R_{L C} /\left(2+R_{L C} / R_{O C}\right) \approx 2 R_{C}$ (Tab. 1). Next, we computed the flowrate $Q$ associated with each branch of the circuit when a total $10^{5} \mathrm{~Pa}$ pressure difference was imposed between the inlet and outlet reservoirs (Tab. 2). In particular, for the S-setup one has $Q_{O C}=Q_{S} /\left(1+2 R_{O C} / R_{L C}\right) \approx Q_{S} R_{L C} / 2 R_{O C}$. Finally, flow velocities and advection times were roughly derived as $U=Q / S, S$ being the cross-section of the channel, and $t_{a d v}=L / U$ (Tab. 2).

On the other hand, the time it takes for a chemical species to fill the observation chamber by diffusion is given by $t_{\text {dif }}=L^{2} / \mathcal{D}$, with $D$ the diffusion coefficient of a typical small inorganic ion, i.e. $10^{-9} \mathrm{~m}^{2} / \mathrm{s}$ [8]. 


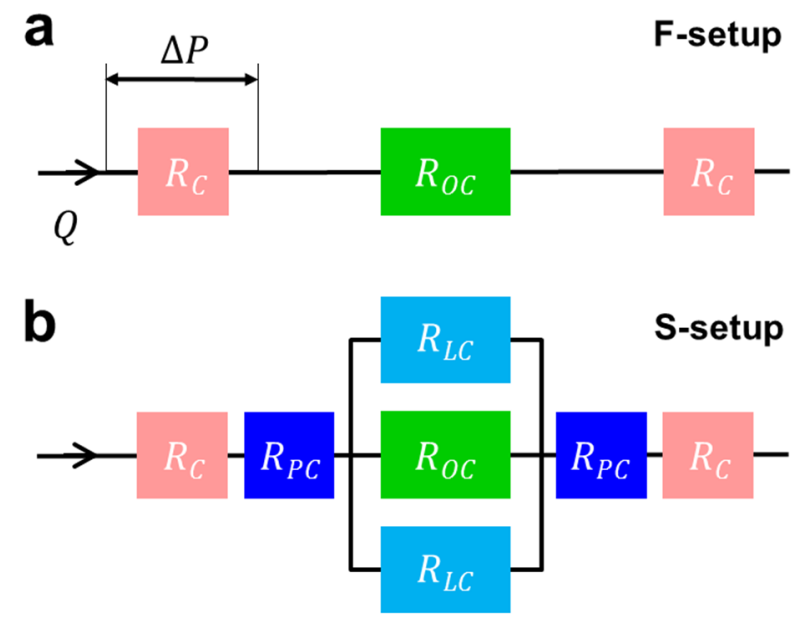

Figure 3: Hydrodynamic diagrams corresponding to the two fluidic setups of Fig. 2. (a) The fully closed chamber setup. (b) The semi-closed chamber setup. $C$ stands for capillary, OC for observation chamber, PC for portal cavity, and LC for lateral cavity.
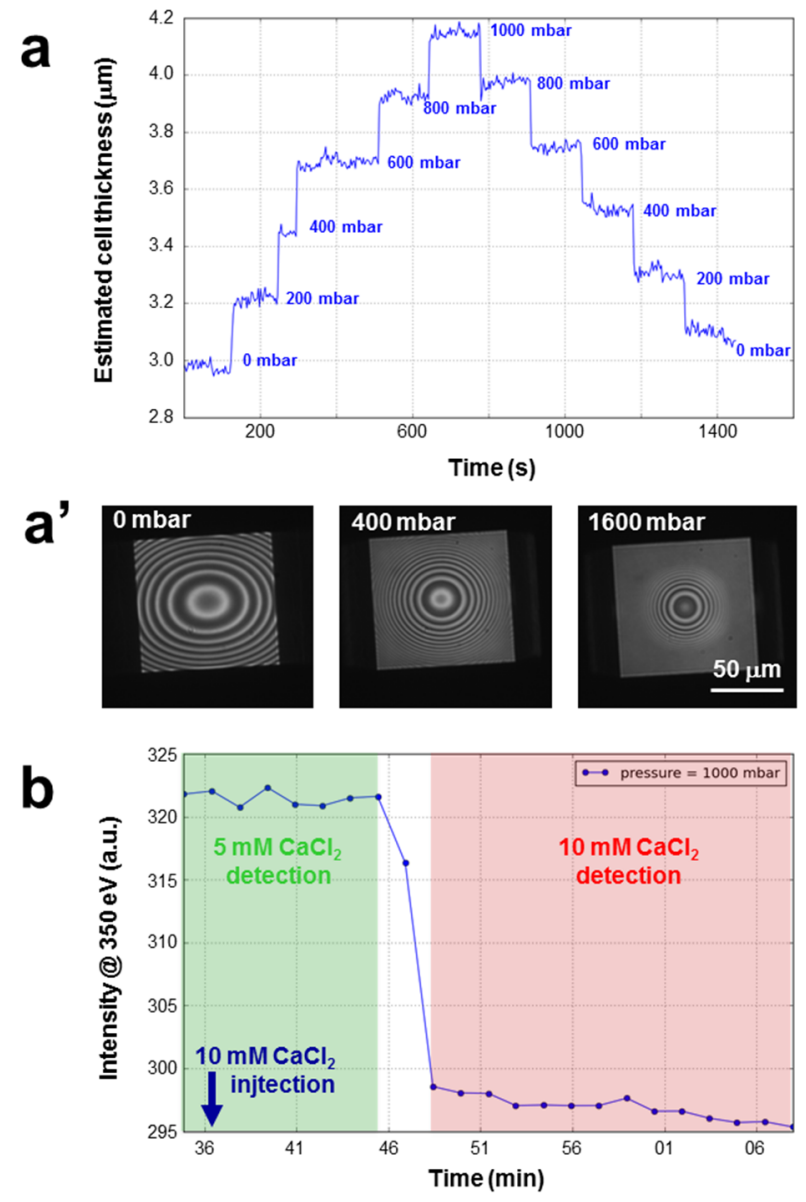

Figure 4: Hydrodynamic characterization of the fluidic cell mounted on the HERMES STXM. (a) Bulging of the SiN membranes upon application of various hydrodynamic pressures. The water layer thickness was determined using the linear attenuation coefficient of water at $510 \mathrm{eV}$ and the Beer-Lambert law. (a') Same experiment followed on a benchtop optical microscope. (b) Liquid exchange time-course observed thanks to calcium absorption.
Table 1: Dimensions and hydrodynamic resistances for the various compartments used in the Fig. 3 "resistor models", as well as for the two setups.

\begin{tabular}{lcccl}
\hline & $\begin{array}{c}W \text { or } D \\
\left(10^{-3} \mathrm{~m}\right)\end{array}$ & $\begin{array}{c}H \\
\left(10^{-3} \mathrm{~m}\right)\end{array}$ & $\begin{array}{c}L \\
\left(10^{-3} \mathrm{~m}\right)\end{array}$ & $\begin{array}{c}R \\
\left(\mathrm{~Pa} . \mathrm{s} / \mathrm{m}^{3}\right)\end{array}$ \\
\hline \multicolumn{2}{c}{ Compartment } & & & \\
$\mathrm{C}$ & 0.25 & & 800 & $8.34 \times 10^{12}$ \\
$\mathrm{PC}$ & 5 & 0.4 & 1 & $3.95 \times 10^{7}$ \\
$\mathrm{LC}$ & 1 & 0.4 & 5 & $1.25 \times 10^{9}$ \\
OC & 1.5 & 0.003 & 5 & $1.48 \times 10^{15}$ \\
$\quad$ Whole setup & & & \\
$\mathrm{F}$ & & & & $1.50 \times 10^{15}$ \\
$\mathrm{~S}$ & & & & $1.67 \times 10^{13}$ \\
\hline
\end{tabular}

\section{RESULTS}

\section{Flow control}

We applied up to 2.5 bars in the reagents reservoir while the outlet was kept at atmospheric pressure. It yielded a flow rate up to $6 \mu \mathrm{L} / \mathrm{min}$, in agreement with the value of the hydrodynamic resistance given for the Fsetup in Table 1. The pressure difference across the $\mathrm{SiN}$ membranes was thus up to 1.75 bar, which induced their bulging (Fig. 4a-a') but no rupture. Any change in the applied pressure resulted in a response at the membrane within seconds, a stable regime being then readily reached (Fig. 4a). Safe operation could take place for $12 \mathrm{~h}$ without leak, as attested by equal flow rates measured at both inlet and outlet, as well as by a stable pressure in the microscope chamber.

Switching the injected solution resulted in a change in X-ray absorption $\sim 10$ min later (Fig. 3b), in agreement with the values of the advection time reported for the capillary and for the F-setup in Table 2.

Table 2: Transport times associated with the input capillary and with the observation chamber when a $10^{5}$ Pas pressure difference is enforced.

\begin{tabular}{lcccc}
\hline & $\begin{array}{c}Q \\
\left(\mathrm{~m}^{3} / \mathrm{s}\right)\end{array}$ & $\begin{array}{c}U \\
(\mathrm{~m} / \mathrm{s})\end{array}$ & $\begin{array}{c}t_{\text {adv }} \\
(\mathrm{s})\end{array}$ & $\begin{array}{c}t_{\text {dif }} \\
(\mathrm{s})\end{array}$ \\
\hline & $F_{\text {-setup }}$ & & & \\
$\mathrm{C}$ & $6.67 \times 10^{-11}$ & $1.36 \times 10^{-3}$ & $5.89 \times 10^{2}$ & \\
$\mathrm{OC}$ & $6.67 \times 10^{-11}$ & $1.48 \times 10^{-2}$ & $3.37 \times 10^{-1}$ & $2.5 \times 10^{4}$ \\
& $S_{\text {-setup }}$ & & & \\
$\mathrm{C}$ & $6.00 \times 10^{-9}$ & $1.22 \times 10^{-1}$ & 6.55 & \\
OC & $2.53 \times 10^{-15}$ & $5.62 \times 10^{-7}$ & $8.89 \times 10^{3}$ & $2.5 \times 10^{4}$ \\
\hline
\end{tabular}

\section{Precipitation imaging}

Injecting reagents through a capillary is much faster than mounting a new cell. We thus tried to image some intermediates during $\mathrm{CaCO}_{3}$ precipitation, using a biomimetic system in which crystal growth is controlled by synthetic polymers. As evidenced by the shoulder present in the absorption spectra of some nanoparticles, a type 2 amorphous state was detected (Fig. 5) [9]. 

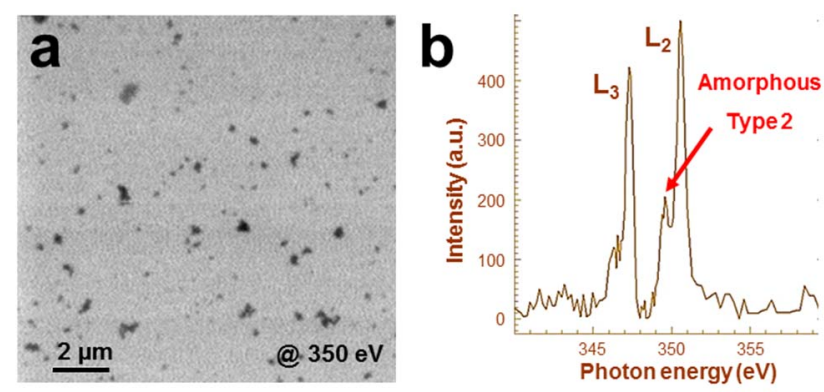

Figure 5: Characterization on the PolLux STXM of solid nanoparticles obtained by precipitation of a $9 \mathrm{mM}$ supersaturated $\mathrm{CaCO}_{3}$ solution containing 500 ppm of sodium polyacrylate. (a) Absorption image obtained at the calcium $L_{2}$ peak. (b) Absorption spectra recorded on a single nanoparticle.

\section{DISCUSSION}

To compare the fully closed chamber with the traditional semi-closed one we evaluated solute transport for both configurations. More precisely, numerical estimations for the latter design were realized for chips having the same characteristics than ours, as well as for dimensions of the surrounding cavities that seemed realistic and in agreement with our knowledge of the various commercial solutions. However, in absence of any experimental investigations, the numbers provided in Table 2 for the S-setup are only indicative.

Data were computed for a 1 bar pressure difference between the inlet and outlet capillaries. This value corresponds to a common limitation imposed by membrane bulging and risk of rupture. For the F-setup the solute exchange rate is limited by a low overall flow rate, a consequence of the high hydrodynamic resistance of the closed and thin observation chamber. Moreover, in the considered pressure regime molecular transport in between the two silicon chips by advection is faster than by diffusion. Conversely, for the S-setup capillary filling is very fast because large hydrodynamic shunts surround the observation chamber. In return, the flow speed at the $\mathrm{SiN}$ windows is low and advection is not significantly more efficient than diffusion to bring reagents under the beam. All in all, the fully closed design seems able to exchange solutes 10 times faster than the semi-closed one. Another advantage is that flow lines are well defined and dead volumes minimum, which ensures a complete control on the nature of the solution present in between the silicon nitride windows.

In the future we plan to install derivation valves close to the fluidic cell in order to speed up the filling of the capillaries, thereby making the advection time in the observation chamber the sole factor limiting kinetic investigations. A temporal resolution of $\sim 1 \mathrm{~s}$ could then possibly be reached. We also expect to apply the conception principles developed in this paper to transmission electron microscopy [10].

\section{ACKNOWLEDGEMENTS}

We thank M. Moskura and P. Haltebourg (CEASaclay, Gif-sur-Yvette), as well as A. Vecchiola (LPNCNRS, Marcoussis), for technical assistance in early experiments, P. Dumas (SOLEIL synchrotron, SaintAubin) for useful discussions, and B. Watts (SLS, Villigen) for help during STXM observations on the PolLux endstation. This work was supported by the grants "FluoBioMix" and "FluoBioMix 2" (RTRA Triangle de la Physique 2009 and 2010), "TEMPOS" (Equipex 2010), "Vermicell" and "Vermicell 2" (Labex NanoSaclay 2012 and 2017), and "Capress" (Cnano IdF 2016).

\section{REFERENCES}

[1] J. Rieger, et al., "Study of precipitation reactions by $\mathrm{X}$-ray microscopy: $\mathrm{CaCO}_{3}$ precipitation and the effect of polycarboxylates", Langmuir, vol. 16, pp. 83008305, 2000.

[2] J. M. Grogan, H. H. Bau, "The nanoaquarium: A platform for in situ transmission electron microscopy in liquid media", J. MEMS, vol. 19, pp. 885-894, 2010.

[3] J. F. Creemer, et al., "A MEMS reactor for atomicscale microscopy of nanomaterials under industrially relevant conditions", J. MEMS, vol. 19, pp. 254-264, 2010.

[4] E. A. Ring, N. de Jonge, "Microfluidic system for transmission electron microscopy", Microsc. Microanal., vol. 16, pp. 622-629, 2010.

[5] M. E. Holtz, et al., "Nanoscale imaging of lithium ion distribution during in situ operation of battery electrode and electrolyte", Nano Lett., vol. 14, pp. 1453-1459, 2014.

[6] H. A. Stone, et al., "Engineering flows in small devices: Microfluidics toward a lab-on-a-chip", Annu. Rev. Fluid Mech., vol. 36, pp. 381-411, 2004.

[7] E. Guyon, et al., Physical Hydrodynamics, Oxford University Press, 2015.

[8] D. R. Lide, Handbook of Chemistry and Physics, CRC Press, 2001.

[9] Y. Politi, et al., "Transformation mechanism of amorphous calcium carbonate into calcite in the sea urchin larval spicule", Proc. Natl. Acad. Sci. USA, vol. 105, 17362-17366, 2008.

[10] F. M. Ross, "Opportunities and challenges in liquid cell electron microscopy", Science, vol. 350, aaa9886, 2015.

\section{CONTACT}

* C. Gosse, tel: +33-(0)1-69-63-61-55;

charlie.gosse@lpn.cnrs.fr 